\title{
Left Ventricular Systolic Function Affects Right Atrial Pressure as Prognosticator in Patients With Heart Failure
}

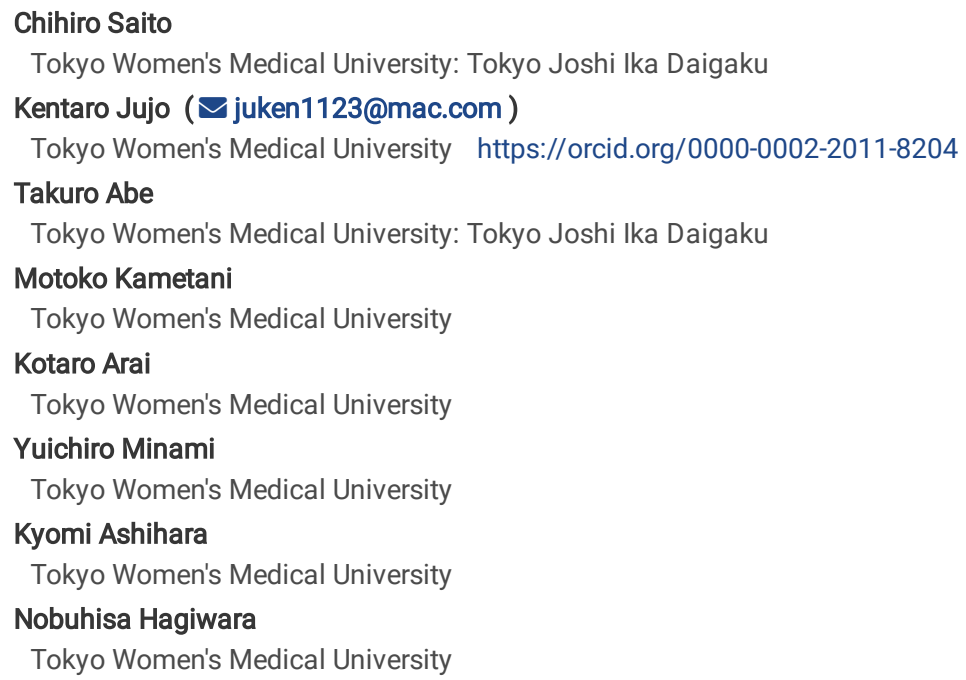

Version of Record: A version of this preprint was published at The International Journal of Cardiovascular Imaging on February 25th, 2022. See the published version at https://doi.org/10.1007/s10554-022-02550-x. 


\section{Abstract \\ BACKGROUND}

The effect of the left ventricular ejection fraction (LVEF) on the prognostic impact of the right atrial pressure (RAP) in patients with heart failure (HF) requires clarification. We aimed to investigate whether LVEF affects the prognostic impact of RAP estimated from inferior vena cava (IVC) measurements in patients hospitalized with HF.

\section{METHODS}

Initially, this observational study included 1,349 consecutive patients urgently hospitalized with HF. After patient exclusions, 506 and 484 patients with reduced $(<40 \%)$ and with non-reduced ( $\geq 40 \%$ ) LVEF, respectively, were assigned according to maximum IVC diameter and its collapsibility, to the Normal-RAP (diameter $\leq 2.1 \mathrm{~cm}$; collapsibility $\geq 50 \%$ ), High-RAP (diameter $>2.1 \mathrm{~cm}$; collapsibility $<50 \%$ ), and Intermediate-RAP (others) groups. The endpoint comprised cardiovascular death after discharge and hospitalization for HF recurrence.

\section{RESULTS}

During the observation period, 247 (49\%) patients with LVEF $<40 \%$ and 178 (37\%) patients with LVEF $\geq 40 \%$ experienced the endpoint. The patient subgroups with LVEF $<40 \%$ had comparable event rates $\left(p_{\text {trend }}=0.10\right.$ ). The High-RAP subgroup with LVEF $\geq 40 \%$ had a higher event rate than the other subgroups $(p<0.001)$. The RAP independently predicted the endpoint in patients with LVEF $\geq 40 \%$ (hazard ratio: 1.26; 95\% confidence interval: 1.01-1.59). The interaction between the RAP groups and LVEF regarding the primary endpoint was significant $\left(p_{\text {interaction }}=0.007\right)$.

\section{CONCLUSION}

Stratifying patients with HF according to IVC measurements may predict the post-discharge cardiovascular prognoses of patients with non-reduced LVEF, but not that of patients with reduced LVEF.

\section{Condensed Abstract}

This observational study of patients discharged after hospitalization for heart failure (HF) comprised 506 with reduced ( $<40 \%)$ and 484 with non-reduced $(\geq 40 \%)$ left ventricular ejection fractions (LVEF). Each patient was assigned to a normal-, intermediate-, or high-right atrial pressure (RAP) group, based on the maximum inferior vena cava diameter and its collapsibility. Multivariate analyses revealed that the RAP independently predicted the composite endpoint of cardiovascular death after discharge and rehospitalization for HF recurrence in patients with non-reduced LVEF, but not in those with reduced LVEF.

\section{Introduction}

Heart failure (HF) is a common and leading cause of cardiovascular morbidity and hospitalization, especially among elderly patients ${ }^{1}$. Mortality rates after discharge do not differ between patients with HF and preserved left ventricular ejection fractions (LVEF) (HFpEF) and those with HF and reduced LVEF (HFrEF)

23 . Furthermore, therapies that reduce the mortality or rehospitalization rates of patients with HFpEF have not been approved; effective therapies and prognostic factors for this population are topics of discussion 45 .

Bedside echocardiography that evaluates the inferior vena cava (IVC) is a noninvasive, reproducible, and feasible means of estimating the right atrial pressure (RAP) ${ }^{6}$. A combination of IVC diameter and the presence of inspiratory collapse correlates strongly with the RAP, estimates hemodynamic congestion, and it is a noninvasive surrogate marker for the systemic fluid volume ${ }^{7}$. A higher degree of congestion is associated with a worse clinical prognosis for patients with acute $\mathrm{HF}^{8}$. IVC assessments at discharge might more effectively identify subclinical volume overloads and predict rehospitalization as a consequence of HF (HHF) with a predominantly ischemic etiology ${ }^{9}$. However, the effect of left ventricular systolic function on the clinical efficacy of RAP estimates based on IVC parameters among patients hospitalized with HF has not been evaluated comprehensively. Hence, this study aimed to investigate whether the LVEF affects the prognostic impact of RAP evaluations based on IVC measurements in patients hospitalized with HF.

\section{Methods}

STUDY POPULATION AND ENDPOINTS. This observational study was undertaken at a single cardiovascular center, and it initially included 1,349 consecutive patients who were hospitalized urgently as a consequence of acute decompensated HF between July 2013 and November 2017. The patients were diagnosed with HF using the Framingham HF diagnostic criteria ${ }^{10}$. After excluding the patients who underwent regular hemodialysis, died in hospital, and lacked data describing their IVC measurements during the index hospitalization, 990 patients, comprising 506 patients with reduced (<40\%) and 484 with non-reduced ( $\geq$ 40\%) LVEF were ultimately enrolled in this study. Using the American Society of Echocardiography guidelines ${ }^{6}$, the patients with reduced and non-reduced LVEF were assigned to 3 groups that were defined according to the maximum IVC diameter and its collapsibility, namely, the Normal-RAP group (IVC diameter: $\leq 2.1 \mathrm{~cm}$; collapsibility $\geq 50 \%$ ), High-RAP group (IVC diameter: $>2.1 \mathrm{~cm}$; collapsibility < 50\%), and Intermediate-RAP group (others). Representative echocardiographic images of patients with the Normal-RAP group (IVC diameter: $\leq 2.1 \mathrm{~cm}$; collapsibility $\geq 50 \%$ ) and High-RAP group (IVC diameter: > $2.1 \mathrm{~cm}$; collapsibility $<50 \%$ ) are shown in Fig. 1 . We compared the groups with reduced and non-reduced LVEF in relation to their clinical data, laboratory data at 
discharge, medications at discharge, and long-term prognoses after discharge. The study's endpoint was a composite of cardiovascular death after discharge and HHF.

The study's protocol was approved by the ethics committee at Tokyo Women's Medical University, and patient enrollment was carried out according to the principles of the Declaration of Helsinki. Written informed consent was obtained only for the use of data from the patients' medical records before study enrollment.

ECHOCARDIOGRAPHY. A comprehensive transthoracic echocardiography (TTE) study, including 2-dimensional, M-mode, Doppler echocardiography, and tissue Doppler imaging, was performed according to the American Society of Echocardiography guidelines ${ }^{11}$. The TTE examinations were performed using a Vivid 7 (GE Healthcare, Horten, Norway) or an iE33 (Philips Healthcare, Bothell, WA, USA) ultrasound system. The TTE evaluations were performed on the patients before they were discharged from their index hospitalizations ${ }^{12}$. The LVEF was calculated using a modification of Simpson's method. To assess the mitral inflow, the E wave velocity was measured using pulsed-wave Doppler imaging, and e' represented the septal mitral annular diastolic velocity measured using tissue Doppler imaging. The tricuspid annular plane systolic excursion (TAPSE) was measured using M-mode echocardiography with the cursor optimally aligned along the direction of the tricuspid lateral annulus in the apical 4-chamber view. The pulmonary artery systolic pressure (PASP) was estimated from the maximal tricuspid regurgitation jet velocity, and the RAP was estimated from the response of IVC to a sniff test. The diameter of IVC was measured in the subcostal long-axis view perpendicular to IVC with the patient in the supine position at $1.0-2.0 \mathrm{~cm}$ from the junction with the right atrium. Inspiratory collapse is expressed as the IVC collapsibility index, which is calculated as (maximum IVC diameter - minimum IVC diameter)/maximum IVC diameter $\times 100$.

STATISTICAL ANALYSES. The Kruskal-Wallis test was used to compare the groups regarding the skewed continuous variables, and the chi-squared test was used to compare the groups regarding the categorical variables. Cardiovascular death after discharge and HHF were evaluated using Kaplan-Meier curves, and differences among the groups were assessed by using log-rank tests for trends. Univariate Cox proportional hazards regression analyses were performed to evaluate associations between the RAP classifications, which were defined according to the IVC measurements, and the prognoses after discharge in each group including patients with reduced and non-reduced LVEF. Variables were included in the multivariable model if they reached a level of significance of $p<$ 0.05 in the univariate analyses and they were considered clinically significant. Multivariable Cox proportional hazards regression analyses were performed to exclude the confounding factors and identify independent risk factors associated with the composite endpoint of cardiovascular death and HHF, and cardiovascular death and HHF, respectively. The interaction between the RAP groups and LVEF regarding the composite endpoint of cardiovascular death and HHF was assessed using the respective statistical model. A 2-sided value of $p<0.05$ was considered statistically significant. The statistical analyses were performed using R software, version 3.3.0 (R Foundation for Statistical Computing, Vienna, Austria).

\section{Results}

Of the 506 reduced LVEF patients, 177 (35\%) were assigned to the Normal-RAP, 239 (47\%) were assigned to the Intermediate-RAP, and 90 (18\%) were assigned to the High-RAP groups. Of the 484 non-reduced LVEF patients, 147 (30\%) were assigned to the Normal-RAP, 221 (46\%) were assigned to the Intermediate-RAP, and 116 (24\%) were assigned to the High-RAP groups (Reduced vs. Non-reduced LVEF: $p=0.044$ ).

CLINICAL CHARACTERISTICS. Table 1 shows the patients' baseline characteristics. Overall, the patients' mean age was 69 years and two-thirds of the patients were men. The High-RAP group had a significantly lower rate of dyslipidemia, higher rate of atrial fibrillation, and lower systolic blood pressure at discharge compared with the other RAP groups. The laboratory data at discharge showed that the High-RAP group had a lower estimated glomerular filtration rate (eGFR), a higher blood urea nitrogen (BUN) level, and lower hemoglobin and sodium levels, and that the Intermediate-RAP group had a higher BNP level than the other RAP groups. The TTE findings showed that the High-RAP groups had higher LVEF and lower TAPSEs than the other RAP groups, and that as the RAP increased, the PASP increased. Almost $90 \%$ of the enrolled patients received furosemide. The High-RAP group was prescribed furosemide more frequently, and beta-blockers and statins less frequently than the other RAP groups. The Normal-RAP group was prescribed mineralocorticoid receptor antagonists less frequently than the other RAP groups. 
Table 1

Patients' baseline characteristics

\begin{tabular}{|c|c|c|c|c|c|c|c|c|c|c|c|}
\hline \multirow[t]{3}{*}{ Variables } & \multicolumn{4}{|l|}{ All } & \multicolumn{4}{|c|}{ educed LVEF } & \multicolumn{3}{|c|}{ Non-reduced LVEF } \\
\hline & $\begin{array}{l}\text { Normal } \\
\text { RAP }\end{array}$ & $\begin{array}{l}\text { Intermediate } \\
\text { RAP }\end{array}$ & $\begin{array}{l}\text { High } \\
\text { RAP }\end{array}$ & $\mathrm{p}_{\text {Value }}$ & $\begin{array}{l}\text { Normal } \\
\text { RAP }\end{array}$ & $\begin{array}{l}\text { Intermediate } \\
\text { RAP }\end{array}$ & $\begin{array}{l}\text { High } \\
\text { RAP }\end{array}$ & $\mathrm{p}_{\text {Value }}$ & $\begin{array}{l}\text { Normal } \\
\text { RAP }\end{array}$ & $\begin{array}{l}\text { Intermediate } \\
\text { RAP }\end{array}$ & $\begin{array}{l}\text { High } \\
\text { RAP }\end{array}$ \\
\hline & $\begin{array}{l}N= \\
324\end{array}$ & $N=460$ & $\begin{array}{l}N= \\
90\end{array}$ & & $N=177$ & $N=239$ & $\begin{array}{l}N= \\
90\end{array}$ & & $N=147$ & $N=221$ & $\begin{array}{l}N= \\
116\end{array}$ \\
\hline \multicolumn{12}{|l|}{$\begin{array}{l}\text { Clinical } \\
\text { background }\end{array}$} \\
\hline Age, years old & $\begin{array}{l}69.4 \pm \\
15.3\end{array}$ & $69.2 \pm 15.4$ & $\begin{array}{l}72.2 \\
\pm \\
12.9\end{array}$ & 0.04 & $\begin{array}{l}65.8 \pm \\
14.7\end{array}$ & $65.1 \pm 16.4$ & $\begin{array}{l}67.7 \\
\pm \\
15.3\end{array}$ & 0.39 & $\begin{array}{l}73.7 \pm \\
14.9\end{array}$ & $73.7 \pm 12.7$ & $\begin{array}{l}75.7 \\
\pm 9.4\end{array}$ \\
\hline Male & $\begin{array}{l}202 \\
(62 \%)\end{array}$ & $288(63 \%)$ & $\begin{array}{l}147 \\
(71 \%)\end{array}$ & 0.06 & $\begin{array}{l}130 \\
(73 \%)\end{array}$ & $179(75 \%)$ & $\begin{array}{l}76 \\
(84 \%)\end{array}$ & 0.11 & $\begin{array}{l}72 \\
(49 \%)\end{array}$ & $109(49 \%)$ & $\begin{array}{l}71 \\
(61 \%)\end{array}$ \\
\hline $\mathrm{BMI}, \mathrm{kg} / \mathrm{m}^{2}$ & $\begin{array}{l}22.5 \pm \\
4.6\end{array}$ & $21.6 \pm 4.5$ & $\begin{array}{l}21.9 \\
\pm 3.1\end{array}$ & 0.10 & $\begin{array}{l}22.5 \pm \\
4.6\end{array}$ & $21.6 \pm 4.5$ & $\begin{array}{l}21.9 \\
\pm 3.1\end{array}$ & 0.10 & $\begin{array}{l}22.4 \pm \\
4.7\end{array}$ & $21.8 \pm 4.0$ & $\begin{array}{l}21.1 \\
\pm 4.0\end{array}$ \\
\hline Hypertension & $\begin{array}{l}222 \\
(69 \%)\end{array}$ & $293(64 \%)$ & $\begin{array}{l}121 \\
(59 \%)\end{array}$ & 0.07 & $\begin{array}{l}118 \\
(67 \%)\end{array}$ & $140(59 \%)$ & $\begin{array}{l}56 \\
(62 \%)\end{array}$ & 0.25 & $\begin{array}{l}104 \\
(71 \%)\end{array}$ & $153(69 \%)$ & $\begin{array}{l}65 \\
(56 \%)\end{array}$ \\
\hline Diabetes & $\begin{array}{l}127 \\
(39 \%)\end{array}$ & $176(38 \%)$ & $\begin{array}{l}83 \\
(40 \%)\end{array}$ & 0.88 & $\begin{array}{l}73 \\
(41 \%)\end{array}$ & $90(38 \%)$ & $\begin{array}{l}35 \\
(39 \%)\end{array}$ & 0.77 & $\begin{array}{l}54 \\
(37 \%)\end{array}$ & $86(38 \%)$ & $\begin{array}{l}48 \\
(41 \%)\end{array}$ \\
\hline Dyslipidemia & $\begin{array}{l}174 \\
(54 \%)\end{array}$ & $234(51 \%)$ & $\begin{array}{l}86 \\
(42 \%)\end{array}$ & 0.02 & $\begin{array}{l}111 \\
(63 \%)\end{array}$ & $129(54 \%)$ & $\begin{array}{l}45 \\
(50 \%)\end{array}$ & 0.09 & $\begin{array}{l}63 \\
(43 \%)\end{array}$ & $105(48 \%)$ & $\begin{array}{l}41 \\
(35 \%)\end{array}$ \\
\hline $\begin{array}{l}\text { Prior } \\
\text { revascularization }\end{array}$ & $\begin{array}{l}74 \\
(23 \%)\end{array}$ & $108(24 \%)$ & $\begin{array}{l}33 \\
(16 \%)\end{array}$ & 0.08 & $\begin{array}{l}41 \\
(23 \%)\end{array}$ & $65(27 \%)$ & $\begin{array}{l}24 \\
(27 \%)\end{array}$ & 0.63 & $\begin{array}{l}33 \\
(22 \%)\end{array}$ & $43(20 \%)$ & $\begin{array}{l}9 \\
(8 \%)\end{array}$ \\
\hline Atrial fibrillation & $\begin{array}{l}139 \\
(43 \%)\end{array}$ & $214(49 \%)$ & $\begin{array}{l}148 \\
(72 \%)\end{array}$ & $<.001$ & $\begin{array}{l}69 \\
(40 \%)\end{array}$ & $89(48 \%)$ & $\begin{array}{l}58 \\
(64 \%)\end{array}$ & $<0.001$ & $\begin{array}{l}70 \\
(48 \%)\end{array}$ & $125(57 \%)$ & $\begin{array}{l}90 \\
(78 \%)\end{array}$ \\
\hline \multicolumn{12}{|c|}{ Hemodynamics at discharge } \\
\hline Systolic BP, mmHg & $\begin{array}{l}113.9 \\
\pm 17.7\end{array}$ & $111.9 \pm 17.8$ & $\begin{array}{l}108.9 \\
\pm \\
16.1\end{array}$ & 0.006 & $\begin{array}{l}109.9 \\
\pm 17.7\end{array}$ & $107.7 \pm 18.5$ & $\begin{array}{l}103.9 \\
\pm \\
16.1\end{array}$ & 0.03 & $\begin{array}{l}118.9 \\
\pm 16.5\end{array}$ & $109.4 \pm 21.3$ & $\begin{array}{l}103.7 \\
\pm \\
14.0\end{array}$ \\
\hline Heart rate, bpm & $\begin{array}{l}69.4 \pm \\
11.5\end{array}$ & $79.6 \pm 12.9$ & $\begin{array}{l}71.1 \\
\pm 12\end{array}$ & 0.24 & $\begin{array}{l}69.5 \pm \\
10.5\end{array}$ & $71.7 \pm 13.2$ & $\begin{array}{l}70.3 \\
\pm \\
10.7\end{array}$ & 0.18 & $\begin{array}{l}69.3 \pm \\
12.7\end{array}$ & $70.3 \pm 10.7$ & $\begin{array}{l}73.0 \\
\pm \\
11.6\end{array}$ \\
\hline \multicolumn{12}{|l|}{$\begin{array}{l}\text { Lab data at } \\
\text { discharge }\end{array}$} \\
\hline Creatinine, $\mathrm{mg} / \mathrm{dL}$ & $\begin{array}{l}1.35 \pm \\
1.06\end{array}$ & $1.49 \pm 1.50$ & $\begin{array}{l}1.50 \\
\pm \\
0.91\end{array}$ & 0.29 & $\begin{array}{l}1.33 \pm \\
0.88\end{array}$ & $1.46 \pm 1.37$ & $\begin{array}{l}1.63 \\
\pm \\
0.98\end{array}$ & 0.14 & $\begin{array}{l}1.37 \pm \\
1.24\end{array}$ & $1.51 \pm 1.62$ & $\begin{array}{l}1.40 \\
\pm \\
0.84\end{array}$ \\
\hline $\begin{array}{l}\text { eGFR, } \\
\mathrm{mL} / \mathrm{min} / 1.73 \mathrm{~m}^{2}\end{array}$ & $\begin{array}{l}48.7 \pm \\
28.0\end{array}$ & $49.1 \pm 40.7$ & $\begin{array}{l}40.8 \\
\pm \\
25.4\end{array}$ & 0.01 & $\begin{array}{l}46.3 \pm \\
26.5\end{array}$ & $48.8 \pm 47.6$ & $\begin{array}{l}35.0 \\
\pm \\
18.2\end{array}$ & 0.01 & $\begin{array}{l}51.5 \pm \\
29.6\end{array}$ & $49.5 \pm 32.2$ & $\begin{array}{l}45.4 \\
\pm \\
29.1\end{array}$ \\
\hline $\mathrm{BUN}, \mathrm{mg} / \mathrm{dL}$ & $\begin{array}{l}27.3 \pm \\
15.4\end{array}$ & $28.1 \pm 15.6$ & $\begin{array}{l}36.9 \\
\pm \\
21.4\end{array}$ & $\begin{array}{l}< \\
0.001\end{array}$ & $\begin{array}{l}26.7 \pm \\
14.7\end{array}$ & $27.5 \pm 15.8$ & $\begin{array}{l}36.9 \\
\pm \\
23.0\end{array}$ & $<.001$ & $\begin{array}{l}27.8 \pm \\
16.3\end{array}$ & $28.7 \pm 19.2$ & $\begin{array}{l}36.8 \\
\pm \\
20.2\end{array}$ \\
\hline Albumin, $\mathrm{mg} / \mathrm{dL}$ & $\begin{array}{l}3.67 \pm \\
0.52\end{array}$ & $3.65 \pm 0.54$ & $\begin{array}{l}3.70 \\
\pm \\
0.53\end{array}$ & 0.51 & $\begin{array}{l}3.76 \pm \\
0.48\end{array}$ & $3.68 \pm 0.53$ & $\begin{array}{l}3.76 \\
\pm \\
0.50\end{array}$ & 0.24 & $\begin{array}{l}3.54 \pm \\
0.55\end{array}$ & $3.62 \pm 0.55$ & $\begin{array}{l}3.66 \\
\pm \\
0.54\end{array}$ \\
\hline $\begin{array}{l}\text { Hemoglobin, } \\
\text { mg/dL }\end{array}$ & $\begin{array}{l}12.5 \pm \\
2.1\end{array}$ & $12.2 \pm 2.1$ & $\begin{array}{l}11.5 \\
\pm 2.3\end{array}$ & $\begin{array}{l}< \\
0.001\end{array}$ & $\begin{array}{l}12.9 \pm \\
2.1\end{array}$ & $12.7 \pm 2.1$ & $\begin{array}{l}12.3 \\
\pm 2.5\end{array}$ & 0.14 & $\begin{array}{l}12.0 \pm \\
2.0\end{array}$ & $11.7 \pm 3.2$ & $\begin{array}{l}10.8 \\
\pm 1.9\end{array}$ \\
\hline Sodium, $\mathrm{mEq} / \mathrm{L}$ & $\begin{array}{l}138.9 \\
\pm 8.3\end{array}$ & $138.8 \pm 3.3$ & $\begin{array}{l}137.3 \\
\pm 3.9\end{array}$ & 0.002 & $\begin{array}{l}138.5 \\
\pm 10.7\end{array}$ & $138.3 \pm 3.4$ & $\begin{array}{l}136.6 \\
\pm 3.9\end{array}$ & 0.09 & $\begin{array}{l}139.4 \\
\pm 3.4\end{array}$ & $139.3 \pm 3.2$ & $\begin{array}{l}137.9 \\
\pm 3.8\end{array}$ \\
\hline BNP, pg/mL & $\begin{array}{l}341 \pm \\
351\end{array}$ & $439 \pm 537$ & $\begin{array}{l}385 \pm \\
501\end{array}$ & 0.048 & $\begin{array}{l}355 \pm \\
371\end{array}$ & $584 \pm 651$ & $\begin{array}{l}561 \pm \\
630\end{array}$ & 0.001 & $\begin{array}{l}323 \pm \\
324\end{array}$ & $306 \pm 360$ & $\begin{array}{l}235 \pm \\
282\end{array}$ \\
\hline $\mathrm{CRP}, \mathrm{mg} / \mathrm{dL}$ & $\begin{array}{l}0.85 \pm \\
1.82\end{array}$ & $0.88 \pm 1.81$ & $\begin{array}{l}0.91 \\
\pm \\
1.83\end{array}$ & 0.92 & $\begin{array}{l}0.78 \pm \\
1.36\end{array}$ & $0.78 \pm 1.88$ & $\begin{array}{l}0.78 \\
\pm \\
1.36\end{array}$ & 0.96 & $\begin{array}{l}0.93 \pm \\
2.28\end{array}$ & $0.95 \pm 1.75$ & $\begin{array}{l}1.02 \\
\pm \\
2.13\end{array}$ \\
\hline
\end{tabular}

Echocardiographic

parameters

$\mathrm{ACEi}=$ angiotensin-converting enzyme inhibitor; $\mathrm{ARB}=$ angiotensin II receptor blocker; $\mathrm{BMI}=$ body mass index; $\mathrm{BNP}=\mathrm{B}$-type natriuretic peptide; $\mathrm{BP}=$ blood pressure; $\mathrm{BUN}$ = blood urea nitrogen; $\mathrm{CRP}=\mathrm{C}$-reactive protein; eGFR = estimated glomerular filtration rate; IVC = inferior vena cava; $\mathrm{LVEF}=$ left ventricular ejec fraction; $\mathrm{PASP}=$ pulmonary artery systolic pressure; RAP = right atrial pressure; TAPSE = tricuspid annular plane systolic excursion. 


\begin{tabular}{|c|c|c|c|c|c|c|c|c|c|c|c|}
\hline \multirow[t]{3}{*}{ Variables } & \multicolumn{4}{|l|}{ All } & \multicolumn{4}{|c|}{ Reduced LVEF } & \multicolumn{3}{|c|}{ Non-reduced LVEF } \\
\hline & $\begin{array}{l}\text { Normal } \\
\text { RAP }\end{array}$ & $\begin{array}{l}\text { Intermediate } \\
\text { RAP }\end{array}$ & $\begin{array}{l}\text { High } \\
\text { RAP }\end{array}$ & $\mathrm{p}_{\text {Value }}$ & $\begin{array}{l}\text { Normal } \\
\text { RAP }\end{array}$ & $\begin{array}{l}\text { Intermediate } \\
\text { RAP }\end{array}$ & $\begin{array}{l}\text { High } \\
\text { RAP }\end{array}$ & Value & $\begin{array}{l}\text { Normal } \\
\text { RAP }\end{array}$ & $\begin{array}{l}\text { Intermediate } \\
\text { RAP }\end{array}$ & $\begin{array}{l}\text { High } \\
\text { RAP }\end{array}$ \\
\hline & $\begin{array}{l}N= \\
324\end{array}$ & $N=460$ & $\begin{array}{l}N= \\
90\end{array}$ & & $N=177$ & $N=239$ & $\begin{array}{l}N= \\
90\end{array}$ & & $N=147$ & $N=221$ & $\begin{array}{l}N= \\
116\end{array}$ \\
\hline LVEF, \% & $\begin{array}{l}38.7 \pm \\
12.0\end{array}$ & $38.5 \pm 13.0$ & $\begin{array}{l}41.3 \\
\pm \\
13.6\end{array}$ & 0.02 & $\begin{array}{l}29.4 \pm \\
6.4\end{array}$ & $27.8 \pm 7.0$ & $\begin{array}{l}27.9 \\
\pm 6.8\end{array}$ & 0.04 & $\begin{array}{l}49.9 \pm \\
6.4\end{array}$ & $49.9 \pm 6.5$ & $\begin{array}{l}51.8 \\
\pm 6.3\end{array}$ \\
\hline E/e' (septal) & $\begin{array}{l}17.4 \pm \\
7.8\end{array}$ & $18.4 \pm 9.0$ & $\begin{array}{l}18.7 \\
\pm 8.2\end{array}$ & 0.24 & $\begin{array}{l}17.7 \pm \\
8.4\end{array}$ & $19.5 \pm 9.1$ & $\begin{array}{l}20.3 \\
\pm 8.3\end{array}$ & 0.07 & $\begin{array}{l}17.1 \pm \\
7.1\end{array}$ & $17.0 \pm 8.6$ & $\begin{array}{l}17.2 \\
\pm 8.0\end{array}$ \\
\hline TAPSE, mm & $\begin{array}{l}16.9 \pm \\
4.8\end{array}$ & $16.3 \pm 5.0$ & $\begin{array}{l}15.1 \\
\pm 4.9\end{array}$ & 0.004 & $\begin{array}{l}15.6 \pm \\
4.6\end{array}$ & $14.9 \pm 4.4$ & $\begin{array}{l}13.2 \\
\pm 4.7\end{array}$ & 0.006 & $\begin{array}{l}18.7 \pm \\
4.5\end{array}$ & $18.0 \pm 5.1$ & $\begin{array}{l}16.4 \\
\pm 4.6\end{array}$ \\
\hline PASP, mmHg & $\begin{array}{l}40.0 \pm \\
12.3\end{array}$ & $42.2 \pm 14.1$ & $\begin{array}{l}46.2 \\
\pm \\
15.1\end{array}$ & $<.001$ & $\begin{array}{l}31.3 \pm \\
11.7\end{array}$ & $39.3 \pm 13.8$ & $\begin{array}{l}52.6 \\
\pm \\
15.8\end{array}$ & $\begin{array}{l}< \\
0.001\end{array}$ & $\begin{array}{l}34.2 \pm \\
12.9\end{array}$ & $41.5 \pm 14.2$ & $\begin{array}{l}50.5 \\
\pm \\
14.2\end{array}$ \\
\hline $\begin{array}{l}\text { Maximum IVC, } \\
\mathrm{mm}\end{array}$ & $\begin{array}{l}13.7 \pm \\
3.8\end{array}$ & $16.2 \pm 4.5$ & $\begin{array}{l}25.7 \\
\pm 4.0\end{array}$ & $<.001$ & $\begin{array}{l}13.6 \pm \\
3.7\end{array}$ & $15.7 \pm 4.6$ & $\begin{array}{l}25.5 \\
\pm 3.4\end{array}$ & $<.001$ & $\begin{array}{l}13.8 \pm \\
3.8\end{array}$ & $16.7 \pm 4.5$ & $\begin{array}{l}25.9 \\
\pm 4.5\end{array}$ \\
\hline $\begin{array}{l}\text { IVC collapsibility, } \\
\text { mm }\end{array}$ & $\begin{array}{l}59.0 \pm \\
8.1\end{array}$ & $33.1 \pm 14.8$ & $\begin{array}{l}23.1 \\
\pm \\
13.4\end{array}$ & $<.001$ & $\begin{array}{l}58.6 \pm \\
8.1\end{array}$ & $32.6 \pm 15.6$ & $\begin{array}{l}21.3 \\
\pm \\
14.5\end{array}$ & $<.001$ & $\begin{array}{l}59.4 \pm \\
8.2\end{array}$ & $33.6 \pm 13.9$ & $\begin{array}{l}24.5 \\
\pm \\
12.5\end{array}$ \\
\hline \multicolumn{12}{|c|}{ Medications at discharge } \\
\hline Furosemide & $\begin{array}{l}275 \\
(85 \%)\end{array}$ & $398(87 \%)$ & $\begin{array}{l}192 \\
(93 \%)\end{array}$ & 0.01 & $\begin{array}{l}155 \\
(88 \%)\end{array}$ & $209(87 \%)$ & $\begin{array}{l}80 \\
(89 \%)\end{array}$ & 0.97 & $\begin{array}{l}120 \\
(82 \%)\end{array}$ & $189(86 \%)$ & $\begin{array}{l}112 \\
(97 \%)\end{array}$ \\
\hline ACEi/ARBs & $\begin{array}{l}272 \\
(84 \%)\end{array}$ & $373(81 \%)$ & $\begin{array}{l}169 \\
(82 \%)\end{array}$ & 0.59 & $\begin{array}{l}158 \\
(89 \%)\end{array}$ & 207 (87\%) & $\begin{array}{l}81 \\
(90 \%)\end{array}$ & 0.63 & $\begin{array}{l}114 \\
(78 \%)\end{array}$ & $166(75 \%)$ & $\begin{array}{l}88 \\
(76 \%)\end{array}$ \\
\hline$\beta$-blockers & $\begin{array}{l}257 \\
(79 \%)\end{array}$ & $354(77 \%)$ & $\begin{array}{l}141 \\
(68 \%)\end{array}$ & 0.02 & $\begin{array}{l}159 \\
(90 \%)\end{array}$ & $210(88 \%)$ & $\begin{array}{l}79 \\
(88 \%)\end{array}$ & 0.81 & $\begin{array}{l}98 \\
(67 \%)\end{array}$ & $144(65 \%)$ & $\begin{array}{l}62 \\
(53 \%)\end{array}$ \\
\hline $\begin{array}{l}\text { Mineral corticoid- } \\
\text { receptor } \\
\text { antagonist }\end{array}$ & $\begin{array}{l}175 \\
(54 \%)\end{array}$ & $287(62 \%)$ & $\begin{array}{l}128 \\
(62 \%)\end{array}$ & 0.045 & $\begin{array}{l}108 \\
(61 \%)\end{array}$ & $168(70 \%)$ & $\begin{array}{l}66 \\
(73 \%)\end{array}$ & 0.06 & $\begin{array}{l}67 \\
(46 \%)\end{array}$ & $119(54 \%)$ & $\begin{array}{l}62 \\
(53 \%)\end{array}$ \\
\hline Statin & $\begin{array}{l}167 \\
(52 \%)\end{array}$ & $179(39 \%)$ & $\begin{array}{l}67 \\
(33 \%)\end{array}$ & $\begin{array}{l}< \\
0.001\end{array}$ & $\begin{array}{l}99 \\
(56 \%)\end{array}$ & $104(44 \%)$ & $\begin{array}{l}34 \\
(38 \%)\end{array}$ & 0.007 & $\begin{array}{l}68 \\
(46 \%)\end{array}$ & $75(34 \%)$ & $\begin{array}{l}33 \\
(28 \%)\end{array}$ \\
\hline
\end{tabular}

$\mathrm{ACEi}=$ angiotensin-converting enzyme inhibitor; $\mathrm{ARB}=$ angiotensin II receptor blocker; $\mathrm{BMI}=$ body mass index; $\mathrm{BNP}=\mathrm{B}$-type natriuretic peptide; $\mathrm{BP}=$ blood pressure; BUN = blood urea nitrogen; CRP = C-reactive protein; eGFR = estimated glomerular filtration rate; IVC = inferior vena cava; LVEF = left ventricular ejec fraction; $P A S P=$ pulmonary artery systolic pressure; RAP = right atrial pressure; TAPSE = tricuspid annular plane systolic excursion.

The mean age of the patients with reduced LVEF was 65 years, which was 10 years younger than the mean age of the patients with non-reduced LVEF. The rates of hypertension, diabetes, and dyslipidemia were comparable among the different RAP groups in patients with reduced LVEF. The prevalence of atrial fibrillation increased as the RAP rose in the patients with reduced and non-reduced LVEF. At discharge, the systolic blood pressure and serum sodium levels were lower, BUN levels were higher, and the eGFRs were lower in the High-RAP groups of patients with reduced and non-reduced LVEF compared with the other RAP groups. The Normal-RAP group of patients with reduced LVEF had a lower BNP level than the other RAP groups of patients with reduced LVEF. As the RAP increased, the TAPSE declined and the PASP rose in the patients with reduced and non-reduced LVEF. The RAP groups did not differ regarding the use of betablockers, angiotensin-converting enzyme inhibitors/angiotensin II receptor blockers (ACEi/ARB), and mineralocorticoid receptor antagonists, and the use of statins was lower in the High-RAP group compared with that in the other RAP groups of patients with reduced and non-reduced LVEF. The use of furosemide was comparable among the RAP groups of patients with reduced LVEF. Furosemide use was more frequent in the High-RAP group than that in the other RAP groups of patients with non-reduced LVEF.

PATIENT PROGNOSES: PRIMARY ENDPOINT. During the observation period after discharge (median follow-up duration was 472 days), the primary endpoint had occurred in 425 patients (43\%) overall. The Kaplan-Meier curves showed that the rates of the composite endpoint of cardiovascular death and HHF were higher in the patients with higher RAP (log-rank test for trend: $p<0.001$ ) (Figure 2A). Of the patients with reduced and non-reduced LVEF, 247 (49\%) and 178 (37\%), respectively, experienced the primary endpoint. The Kaplan-Meier analysis showed the RAP groups of patients with reduced LVEF did not differ regarding the incidence of the primary endpoint (log-rank for trend: $p=0.10$ ) (Figure 2B). Among the patients with non-reduced LVEF, the High-RAP group showed a higher event rate compared with that in the other RAP groups $(p<0.001)$ (Figure $2 \mathrm{C})$. The univariate Cox regression analysis of the patients with non-reduced LVEF showed that age, the body mass index, hypertension, atrial fibrillation, eGFR, BUN, hemoglobin, and sodium levels at discharge, the furosemide dose, the use of ACEi/ARB, beta-blockers, and statins, and the RAP classification were related to the incidence of the primary endpoint (Table 2). The multivariate Cox regression analysis that accounted for the covariates, showed that the RAP classification persisted as an independent predictor of the primary endpoint (adjusted hazard ratio [HR]: 1.26; 95\% confidence interval [CI]: 1.01-1.59). The interaction between the RAP groups and the LVEF regarding the primary endpoint was significant $\left(\mathrm{p}_{\text {interaction }}=0.007\right)$. 
Table 2

Cox regression analysis of the primary endpoint in patients with non-reduced LVEF

\begin{tabular}{|c|c|c|c|c|}
\hline \multirow[t]{2}{*}{ Covariants } & \multicolumn{2}{|c|}{ Univariate analysis } & \multicolumn{2}{|c|}{ Multivariate analysis } \\
\hline & $\mathrm{HR}(95 \% \mathrm{Cl})$ & p Value & $\mathrm{HR}(95 \% \mathrm{Cl})$ & p Value \\
\hline Age & $1.03(1.01-1.05)$ & 0.002 & $1.01(0.99-1.02)$ & 0.43 \\
\hline BMI & $0.93(0.89-0.98)$ & 0.007 & $0.99(0.94-1.03)$ & 0.51 \\
\hline Hypertension & $0.63(0.43-0.93)$ & 0.02 & $0.96(0.67-1.37)$ & 0.82 \\
\hline Atrial fibrillation & $1.92(1.25-2.95)$ & 0.003 & $1.59(1.11-2.27)$ & 0.01 \\
\hline BUN & $1.02(1.01-1.03)$ & $<0.001$ & $1.01(1.00-1.02)$ & 0.04 \\
\hline Hemoglobin & $0.85(0.77-0.94)$ & 0.002 & $0.94(0.86-1.02)$ & 0.14 \\
\hline Sodium & $0.92(0.87-0.96)$ & $<0.001$ & $0.96(0.91-1.01)$ & 0.10 \\
\hline Furosemide & $3.82(1.67-8.74)$ & 0.002 & $2.03(0.99-4.13)$ & 0.052 \\
\hline ACEi/ARBs & $0.62(0.42-0.92)$ & 0.02 & $0.52(0.37-0.74)$ & $<0.001$ \\
\hline$\beta$-blockers & $0.67(0.46-0.98)$ & 0.04 & $0.61(0.44-0.856)$ & 0.003 \\
\hline RAP classification & $1.55(1.12-2.01)$ & 0.001 & $1.26(1.01-1.59)$ & 0.04 \\
\hline
\end{tabular}

PATIENT PROGNOSES: CARDIOVASCULAR DEATH AND REHOSPITALIZATION FOR HEART FAILURE. Regarding cardiovascular death, HF caused 51 deaths (74\%), and $16(23 \%)$ sudden cardiac deaths and $3(4 \%)$ strokes occurred in the patients with reduced LVEF. Among the patients with non-reduced LVEF, HF caused 41 deaths (80\%), and 7 (14\%) sudden cardiac deaths, 2 (4\%) strokes, and 1 (2\%) cardiovascular hemorrhage occurred (Supplemental Table 1 ).

Overall, the patients with higher RAP had higher cardiovascular mortality rates (log-rank test for trend: $p<0.001)$ (Figure $3 A$ ). Among the patients with reduced LVEF, the cardiovascular mortality rate was lower in the Normal-RAP group compared with the rates in the Intermediate-RAP and High-RAP groups ( $<<0.001$ ) (Figure 3B). Among the patients with non-reduced LVEF, the cardiovascular mortality rate was higher in the High-RAP group compared with the rates in the Intermediate-RAP and Normal-RAP groups $(p<0.001)$ (Figure $3 C)$.

The univariate Cox regression analysis of the patients with reduced LVEF showed that age, the body mass index, atrial fibrillation, BUN, hemoglobin, and BNP levels at discharge, the TAPSE, and the RAP classification were related to the incidence of cardiovascular death (Table 3). The multivariate Cox regression analysis that accounted for the covariates, showed that the RAP classification persisted as an independent predictor of cardiovascular death (adjusted HR: 1.78; $95 \% \mathrm{Cl}$ : 1.05-2.99). The univariate Cox regression analysis of the patients with non-reduced LVEF, showed that the body mass index, hypertension, BUN and serum sodium levels at discharge, and the RAP classification were related to the incidence of cardiovascular death (Table 3). The multivariate Cox regression analysis that accounted for the covariates, showed that the RAP classification persisted as an independent predictor of cardiovascular death (adjusted HR: 2.33; 95\% Cl: 1.45-3.74). 
Table 3

Cox regression analysis of the cardiovascular death in patients with reduced LVEF

\begin{tabular}{|c|c|c|c|c|}
\hline \multirow[t]{2}{*}{ Covariants } & \multicolumn{2}{|c|}{ Univariate analysis } & \multicolumn{2}{|c|}{ Multivariate analysis } \\
\hline & $\mathrm{HR}(95 \% \mathrm{Cl})$ & p Value & $\mathrm{HR}(95 \% \mathrm{Cl})$ & p Value \\
\hline \multicolumn{5}{|l|}{ Reduced LVEF } \\
\hline Age & $1.03(1.02-1.05)$ & $<0.001$ & $1.02(0.99-1.06)$ & 0.12 \\
\hline BMI & $0.87(0.81-0.93)$ & $<0.001$ & $0.96(0.86-1.07)$ & 0.44 \\
\hline Atrial fibrillation & $2.12(1.32-3.42)$ & 0.002 & $1.68(0.78-3.59)$ & 0.18 \\
\hline BUN & $1.02(1.01-1.03)$ & $<0.001$ & $1.01(0.98-1.04)$ & 0.48 \\
\hline Hemoglobin & $0.78(0.69-0.87)$ & $<0.001$ & $0.84(0.68-1.03)$ & 0.10 \\
\hline Log. BNP & $9.61(4.80-19.2)$ & $<0.001$ & $3.21(1.10-9.36)$ & 0.03 \\
\hline TAPSE & $0.91(0.46-0.98)$ & 0.005 & $0.93(0.86-14.00)$ & 0.06 \\
\hline RAP classification & $1.85(1.35-2.55)$ & $<0.001$ & $1.78(1.05-2.99)$ & 0.03 \\
\hline \multicolumn{5}{|l|}{ Non-reduced LVEF } \\
\hline BMI & $0.80(0.73-0.88)$ & $<0.001$ & $0.85(0.77-0.93)$ & $<0.001$ \\
\hline Hypertension & $0.36(0.21-0.64)$ & $<0.001$ & $0.67(0.37-1.23)$ & 0.20 \\
\hline BUN & $1.02(1.01-1.03)$ & 0.009 & $1.01(0.99-1.03)$ & 0.24 \\
\hline Sodium & $0.90(0.84-0.97)$ & 0.003 & $0.97(0.89-1.06)$ & 0.53 \\
\hline RAP classification & $2.93(1.91-4.47)$ & $<0.001$ & $2.33(1.45-3.74)$ & $<0.001$ \\
\hline
\end{tabular}

The Kaplan-Meier curves showed that the patients with higher RAP had a higher incidence of HHF (log-rank test for trend: $p=0.002)$ (Figure 2D). The HHF rate did not differ among the RAP groups of patients with reduced LVEF ( $p=0.33$ ) (Figure 2E). Among the patients with non-reduced LVEF, the HHF rate was higher in the High-RAP group compared with the rates in the other RAP groups $(p<0.001)$ (Figure 2F). The HHF rate was even worse in the High-RAP group of patients with non-reduced LVEF than that in the High-RAP group of patients with reduced LVEF (2-year event-free survival rate: $46.3 \%$ vs. $56.6 \%$ ). The univariate Cox regression analysis of the patients with non-reduced LVEF showed that the body mass index, hypertension, atrial fibrillation, eGFR, BUN, hemoglobin, and serum sodium levels at discharge, and the RAP classification were related to the HHF rate (Table 4). The multivariate Cox regression analysis that accounted for the covariates, showed that the RAP classification persisted as an independent predictor of HHF (adjusted HR: 1.39; 95\% Cl: 1.09-1.78).

Table 4

Cox regression analysis of the heart failure rehospitalization in patients with non-reduced LVEF

\begin{tabular}{|c|c|c|c|c|}
\hline \multirow[t]{2}{*}{ Covariants } & \multicolumn{2}{|l|}{ Univariate analysis } & \multicolumn{2}{|c|}{ Multivariate analysis } \\
\hline & $\mathrm{HR}(95 \% \mathrm{Cl})$ & p Value & $\mathrm{HR}(95 \% \mathrm{Cl})$ & p Value \\
\hline BMI & $0.99(0.91-0.99)$ & 0.02 & $0.98(0.93-1.02)$ & 0.30 \\
\hline Hypertension & $0.69(0.50-0.94)$ & 0.02 & $0.84(0.59-1.19)$ & 0.32 \\
\hline Atrial fibrillation & $1.66(1.18-2.32)$ & 0.003 & $1.55(1.07-2.25)$ & 0.02 \\
\hline eGFR & $0.99(0.99-0.999)$ & 0.02 & $0.99(0.99-1.00)$ & 0.10 \\
\hline BUN & $1.02(1.01-1.03)$ & $<0.001$ & $1.01(0.99-1.02)$ & 0.45 \\
\hline Hemoglobin & $0.82(0.76-0.90)$ & $<0.001$ & $0.91(0.83-1.00)$ & 0.04 \\
\hline Sodium & $0.93(0.89-0.97)$ & $<0.001$ & $0.96(0.92-1.02)$ & 0.16 \\
\hline RAP classification & $1.60(1.28-1.28)$ & $<0.001$ & $1.39(1.09-1.78)$ & 0.007 \\
\hline
\end{tabular}

\section{Discussion}

The principal finding from this retrospective analysis of patients hospitalized with HF was that a noninvasive and reproducible evaluation of the RAP, which was based on the maximum IVC diameter and its collapsibility, successfully predicted a composite of cardiovascular death and HHF in patients with nonreduced LVEF, but not in patients with reduced LVEF. As far as we are aware, this is the first study to investigate the prognostic impact of the RAP, which was estimated using IVC parameters, in patients with reduced and non-reduced LVEF. 
PROGNOSTIC IMPACT OF THE RIGHT ATRIAL PRESSURE. A residual suboptimal volume overload is difficult to evaluate at discharge in some patients with $\mathrm{HF}$, and if a volume overload is sustained, patients are more likely to experience decompensation shortly after discharge ${ }^{13}$. Therefore, optimal estimations of the systemic volume status at discharge are important to minimize the cardiovascular death and HHF rates. However, combined physical examinations of elevated pressure in the jugular veins, rales, edema, and symptoms, for example, dyspnea, fail to predict HHF, and even skilled clinicians can experience difficulties assessing the volume status based on physical examinations alone ${ }^{14}$. Instead of invasive catheterization, which is the gold standard method for evaluating the RAP, echocardiography can be used to measure IVC diameter and its respiratory collapse noninvasively, and excellent correlations have been demonstrated between these parameters and the RAP ${ }^{15}{ }^{16}$. Left ventricular diastolic and systolic dysfunction increase the left atrial pressure, which transmits back through the pulmonary circulation causing pulmonary hypertension, and if this is combined with pre-existing right ventricular dysfunction, it may further worsen tricuspid regurgitation. Together, all of these stresses increase the RAP and IVC distension ${ }^{17}$. Indeed, quick and easy echocardiographic assessments of IVC can be performed at the bedside by non-specialist physicians, and its interobserver variation is low 618 .

Several studies' findings have shown the prognostic competency of IVC assessments for identifying patients with HF who are at risk of HHF 17 19. Assessing IVC diameter at discharge, rather than on admission, might provide a more accurate insight into a patient's condition after treatment during hospitalization, and it could, theoretically, predict the clinical prognosis more accurately after discharge. Setoguchi et al. reported that the predictors of HHF differed between patients with HFrEF and HFpEF, and that a dilated IVC was a risk factor associated with HHF in patients with HFpEF, but not in patients with HFrEF 20 . Likewise, our study's findings showed that evaluating the RAP based on the maximum IVC diameter and its collapsibility successfully predicted a composite outcome of cardiovascular death and HHF among patients with non-reduced LVEF, but not among patients with reduced LVEF.

RATIONALE FOR THIS STUDY'S RESULTS. To maintain cardiac output in patients with HFrEF, a preload increase shifts the pressure-volume loop to the right ${ }^{21}$; therefore, the cardiac output depends on the preload in some patients with HFrEF. In this study, the RAP estimations did not predict the outcome in the patients with reduced LVEF; this could be explained by excessive dehydration caused by high doses of diuretics resulting in a low RAP. Hence, excessive doses of diuretics may not always be beneficial for patients with HFrEF. In contrast, among patients with HFpEF, a preload increase shifts the pressure-volume loop to the upper right, which induces pulmonary congestion when the blood pressure increases ${ }^{21}$. Several studies' findings have shown that elevated left ventricular filling pressures, diastolic dysfunction, and pulmonary hypertension are highly prevalent among patients with HFpEF, and that they are independent risk factors associated with cardiovascular death or HHF ${ }^{22} 23$. In addition, a recent study's findings revealed that IVC diameter and its collapsibility on echocardiography reliably signified the left ventricular filling pressure in patients with HFpEF ${ }^{24}$. These findings support the current study's results that indicated a lower RAP may be associated with a better prognosis in patients with HFpEF. The optimal RAP might vary among individual patients; therefore, close observation is advised, even after discharge, to titrate diuretics and achieve the best doses during follow-up. Evaluations of the maximum IVC diameter and its collapsibility may reflect subclinical volume overloads and help reduce cardiovascular death and HHF rates after discharge, particularly among patients with HFpEF for whom effective therapies and prognostic factors remain unclear.

STUDY LIMITATIONS. This was a retrospective, single-center study that evaluated data from a small number of patients during a relatively short observation period. The TTE evaluations were performed before discharge, but these were not prespecified; therefore, they varied among the patients, which may have affected the results. Atrial fibrillation could be associated with mitral/tricuspid valve regurgitation, and it might affect a patient's prognosis; however, we did not evaluate the degrees of regurgitation. In the future, large-scale prospective trial focusing on the relationships between left ventricular systolic functions and the clinical outcomes is warranted to confirm the findings of the current study.

\section{Conclusions}

Straightforward risk stratifications of patients hospitalized with HF based on the maximum IVC diameter and its collapsibility may predict cardiovascular mortality and HHF after discharge in patients without left ventricular systolic dysfunction, but not in patients with left ventricular systolic dysfunction. Assessing IVC using easy and safe bedside echocardiography may identify subclinical volume overloads and help to improve clinical outcomes after discharge.

\section{Abbreviations And Acronyms}

eGFR = estimated glomerular filtration rate

$\mathrm{HFpEF}=$ heart failure with preserved ejection fraction

$\mathrm{HFrEF}=$ heart failure with reduced ejection fraction

$\mathrm{HHF}=$ hospitalization as a consequence of heart failure recurrence

IVC = inferior vena cava

LVEF = left ventricular ejection fraction

PASP = pulmonary artery systolic pressure

RAP = right atrial pressure

TAPSE $=$ tricuspid annular plane systolic excursion 
TTE = transthoracic echocardiography

\section{Declarations}

\section{Funding sources}

Not applicable.

\section{Conflict of interest and ethical compliance}

There are no conflicts of interest to declare. This manuscript has not been published or presented elsewhere in part or in entirety, and is not under consideration by another journal. All study participants provided informed consent, and the study design was approved by the appropriate ethics review board.

\section{Acknowledgments}

We would like to thank Editage (www.editage.jp) for English language editing.

\section{References}

1. Chen J, Hsieh AF, Dharmarajan K, Masoudi FA, Krumholz HM (2013) National trends in heart failure hospitalization after acute myocardial infarction for Medicare beneficiaries: 1998-2010. Circulation 128:2577-2584

2. Fonarow GC, Stough WG, Abraham WT, Albert NM, Gheorghiade M, Greenberg BH, O'Connor CM, Sun JL, Yancy CW, Young JB, Investigators O-H (2007) and Hospitals. Characteristics, treatments, and outcomes of patients with preserved systolic function hospitalized for heart failure: a report from the OPTIMIZE-HF Registry. J Am Coll Cardiol 50:768-777

3. Yancy CW, Lopatin M, Stevenson LW, De Marco T, Fonarow GC, Committee ASA, Investigators (2006) Clinical presentation, management, and in-hospital outcomes of patients admitted with acute decompensated heart failure with preserved systolic function: a report from the Acute Decompensated Heart Failure National Registry (ADHERE) Database. J Am Coll Cardiol 47:76-84

4. Ather S, Chan W, Bozkurt B, Aguilar D, Ramasubbu K, Zachariah AA, Wehrens XH, Deswal A (2012) Impact of noncardiac comorbidities on morbidity and mortality in a predominantly male population with heart failure and preserved versus reduced ejection fraction. J Am Coll Cardiol 59:998-1005

5. Kitzman DW (2012) Outcomes in patients with heart failure with preserved ejection fraction: it is more than the heart. J Am Coll Cardiol 59:1006-1007

6. Rudski LG, Lai WW, Afilalo J, Hua L, Handschumacher MD, Chandrasekaran K, Solomon SD, Louie EK, Schiller NB (2010) Guidelines for the echocardiographic assessment of the right heart in adults: a report from the American Society of Echocardiography endorsed by the European Association of Echocardiography, a registered branch of the European Society of Cardiology, and the Canadian Society of Echocardiography. J Am Soc Echocardiogr 23:685-713; quiz 786-8

7. Kircher BJ, Himelman RB, Schiller NB (1990) Noninvasive estimation of right atrial pressure from the inspiratory collapse of the inferior vena cava. Am J Cardiol 66:493-496

8. Drazner MH, Rame JE, Stevenson LW, Dries DL (2001) Prognostic importance of elevated jugular venous pressure and a third heart sound in patients with heart failure. N Engl J Med 345:574-581

9. Carbone F, Bovio M, Rosa GM, Ferrando F, Scarrone A, Murialdo G, Quercioli A, Vuilleumier N, Mach F, Viazzi F, Montecucco F (2014) Inferior vena cava parameters predict re-admission in ischaemic heart failure. Eur $\mathrm{J}$ Clin Invest 44:341-349

10. Steg PG, Joubin L, McCord J, Abraham WT, Hollander JE, Omland T, Mentre F (2005) McCullough PA and Maisel AS. B-type natriuretic peptide and echocardiographic determination of ejection fraction in the diagnosis of congestive heart failure in patients with acute dyspnea. Chest 128:21-29

11. Mitchell C, Rahko PS, Blauwet LA, Canaday B, Finstuen JA, Foster MC, Horton K, Ogunyankin KO, Palma RA, Velazquez EJ (2019) Guidelines for Performing a Comprehensive Transthoracic Echocardiographic Examination in Adults: Recommendations from the American Society of Echocardiography. J Am Soc Echocardiogr 32:1-64

12. Yang LT, Kado Y, Nagata Y, Otani K, Otsuji Y, Takeuchi M (2018) Timing on echocardiography and blood laboratory test is important for future outcome association in hospitalized heart failure patients. J Cardiol 71:71-80

13. Gheorghiade M, Vaduganathan M, Fonarow GC, Bonow RO (2013) Rehospitalization for heart failure: problems and perspectives. J Am Coll Cardiol 61:391-403

14. Wang CS, FitzGerald JM, Schulzer M, Mak E, Ayas NT (2005) Does this dyspneic patient in the emergency department have congestive heart failure? JAMA 294:1944-1956

15. Beigel R, Cercek B, Luo H, Siegel RJ (2013) Noninvasive evaluation of right atrial pressure. J Am Soc Echocardiogr 26:1033-1042

16. Merrer J, De Jonghe B, Golliot F, Lefrant JY, Raffy B, Barre E, Rigaud JP, Casciani D, Misset B, Bosquet C, Outin H, Brun-Buisson C (2001) Nitenberg G and French Catheter Study Group in Intensive C. Complications of femoral and subclavian venous catheterization in critically ill patients: a randomized controlled trial. JAMA 286:700-707

17. Torres D, Cuttitta F, Paterna S, Garofano A, Conti G, Pinto A, Parrinello G (2016) Bed-side inferior vena cava diameter and mean arterial pressure predict long-term mortality in hospitalized patients with heart failure: 36 months of follow-up. Eur J Intern Med 28:80-84

18. Andersen GN, Viset A, Mjolstad OC, Salvesen O, Dalen H, Haugen BO (2014) Feasibility and accuracy of point-of-care pocket-size ultrasonography performed by medical students. BMC Med Educ 14:156 
19. Lee HF, Hsu LA, Chang CJ, Chan YH, Wang CL, Ho WJ, Chu PH (2014) Prognostic significance of dilated inferior vena cava in advanced decompensated heart failure. Int J Cardiovasc Imaging 30:1289-1295

20. Setoguchi M, Hashimoto Y, Sasaoka T, Ashikaga T, Isobe M (2015) Risk factors for rehospitalization in heart failure with preserved ejection fraction compared with reduced ejection fraction. Heart Vessels 30:595-603

21. Komamura K (2013) Similarities and Differences between the Pathogenesis and Pathophysiology of Diastolic and Systolic Heart Failure. Cardiol Res Pract 2013:824135

22. Burke MA, Katz DH, Beussink L, Selvaraj S, Gupta DK, Fox J, Chakrabarti S, Sauer AJ, Rich JD, Freed BH, Shah SJ (2014) Prognostic importance of pathophysiologic markers in patients with heart failure and preserved ejection fraction. Circ Heart Fail 7:288-299

23. Shah AM, Cikes M, Prasad N, Li G, Getchevski S, Claggett B, Rizkala A, Lukashevich I, O'Meara E, Ryan JJ, Shah SJ, Mullens W, Zile MR, Lam CSP, McMurray JJV (2019) Solomon SD and Investigators P-H. Echocardiographic Features of Patients With Heart Failure and Preserved Left Ventricular Ejection Fraction. J Am Coll Cardiol 74:2858-2873

24. Berthelot E, Jourdain P, Bailly MT, Bouchachi A, Gellen B, Rouquette A, Damy T, Herve P, Chemla D, Assayag P (2020) Echocardiographic Evaluation of Left Ventricular Filling Pressure in Patients With Heart Failure With Preserved Ejection Fraction: Usefulness of Inferior Vena Cava Measurements and 2016 EACVI/ASE Recommendations. J Card Fail 26:507-514

\section{Figures}

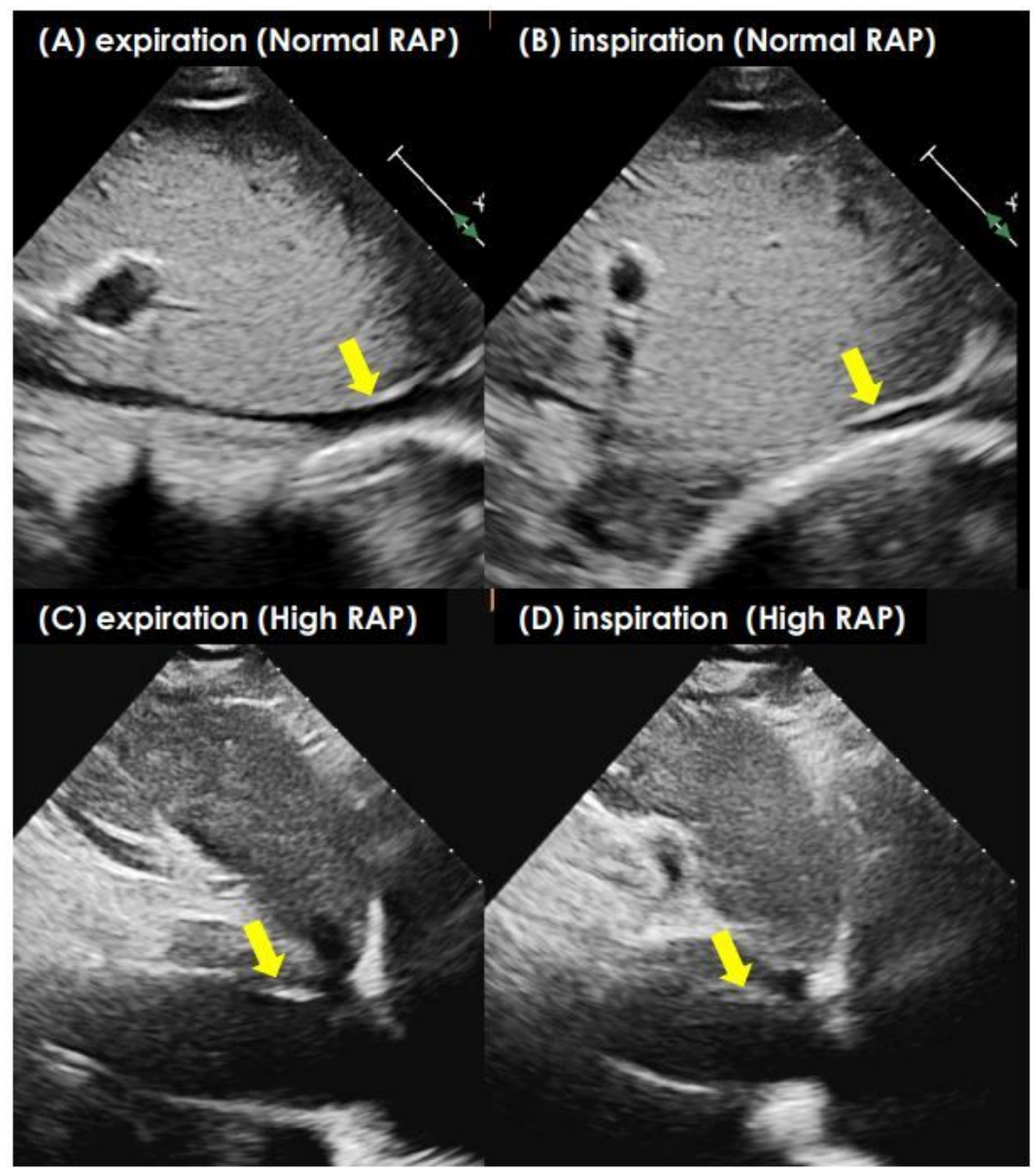

\section{Figure 1}

Representative echocardiographic images of patients with the Normal-RAP group (IVC diameter: $\leq 2.1 \mathrm{~cm}$; collapsibility $\geq 50 \%$ ) and High-RAP group (IVC diameter: $>2.1 \mathrm{~cm}$; collapsibility < 50\%). Subcostal echocardiographic images during (A) expiration (IVC diameter is $8 \mathrm{~mm}$ ) and (B) inspiration (IVC diameter is $2 \mathrm{~mm}$ ) demonstrating good inspiratory collapse of the IVC of a 67-year-old patients in the Normal-RAP group. Echocardiographic images during (C) expiration (IVC diameter is $25 \mathrm{~mm}$ ) and (D) inspiration (IVC diameter is $21 \mathrm{~mm}$ ) demonstrating no inspiratory collapse of the IVC of a 73-year-old patients in the HighRAP group. Yellow-arrow indicates IVC. IVC = inferior vena cava, RAP = right atrial pressure. 


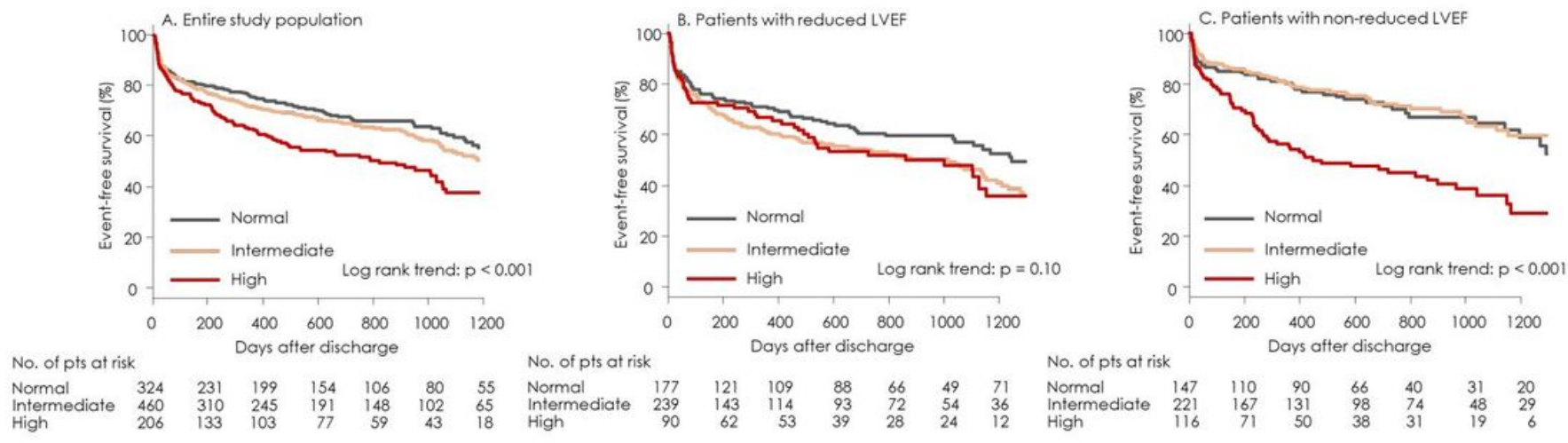

Figure 2

Primary composite endpoint The incidence of a composite of cardiovascular death and rehospitalization as a consequence of heart failure among the patients with normal, intermediate, and high right atrial pressures in the (A) study population overall, (B) patients with reduced left ventricular ejection fractions (LVEF), (C) patients with non-reduced LVEF. LVEF = left ventricular ejection fraction.

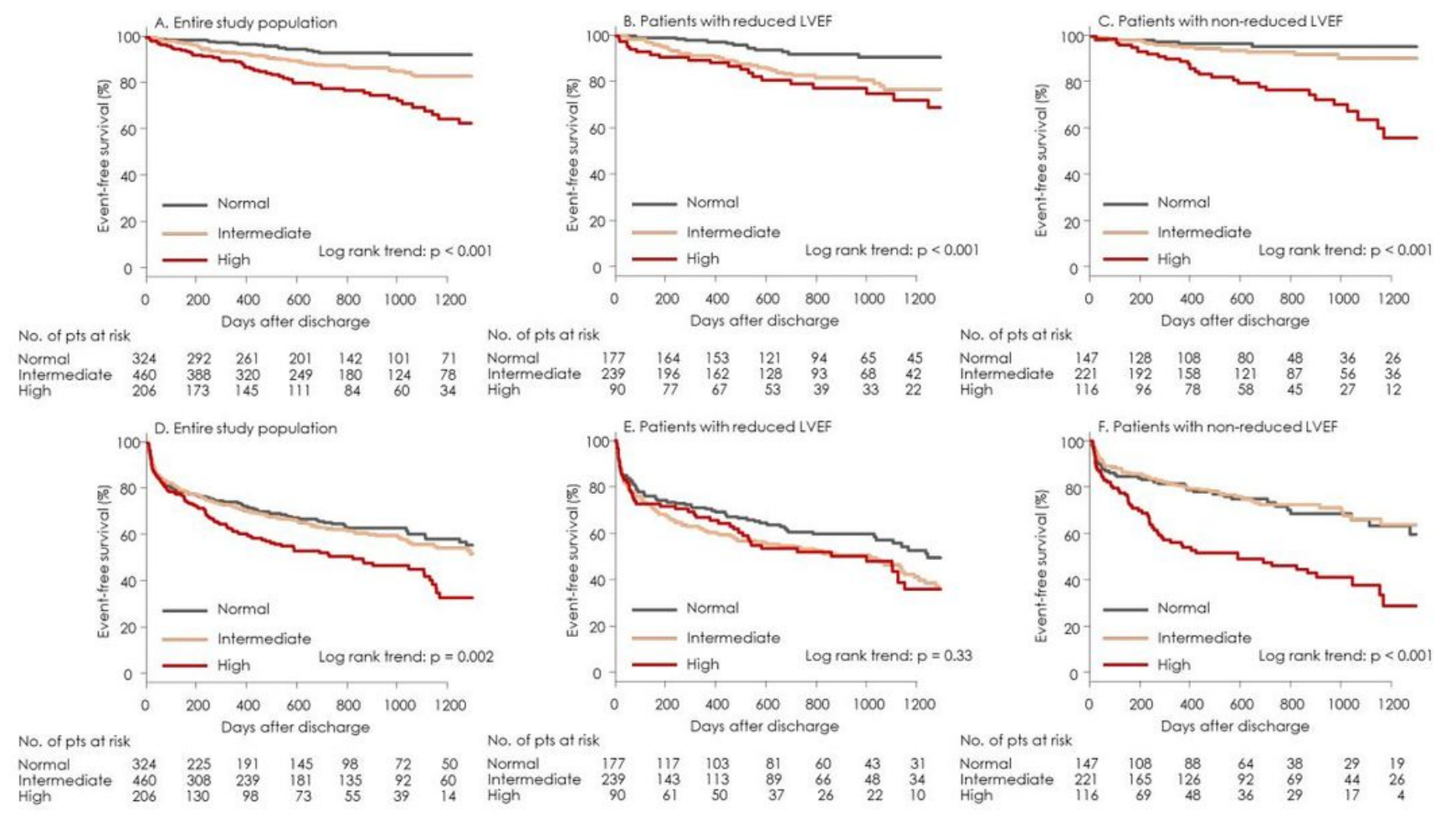

Figure 3

Cardiovascular (CV) death and rehospitalization as a consequence of heart failure (HF) The incidence of cardiovascular death among the patients with normal, intermediate and high right atrial pressures (RAP) in the (A) study population overall, (B) patients with reduced left ventricular ejection fractions (LVEF), and (C) patients with non-reduced LVEF. The incidence of rehospitalization as a consequence of HF among the patients with normal, intermediate, and high RAP in the (D) study population overall, (E) patients with reduced left ventricular ejection fractions (LVEF), and (F) patients non-reduced LVEF. LVEF = left ventricular ejection fraction. 
This is a list of supplementary files associated with this preprint. Click to download.

- SuppTable1IVCHF.docx

Page $12 / 12$ 